\title{
Niementowski reaction: microwave induced and conventional synthesis of quinazolinones and 3-methyl-1H-5-pyrazolones and their antimicrobial activity
}

\author{
Amar R. Desai and Kishor R. Desai* \\ Department of Chemistry, Veer Narmad South Gujarat University, Surat-395007, India \\ E-mail: k_r_desai@rediffmail.com; amardesai1978@yahoo.com
}

(received 20 Feb 05; accepted 15 Apr 05; published on the web 18 Apr 05)

\begin{abstract}
The Niementowski reaction has been extended to synthesize 3-substituted/2,3-disubstituted$4(3 \mathrm{H})$ quinazolinones instead of the 2-substituted derivatives. The methodology is environmentally benign and completely eliminates the need of solvent for the reaction. Neat reactants were cyclocondensed under microwaves to afford, in good yield, the desired product in less irradiation time as compared to the classical technique. The compared results of microwave and conventional techniques have been discussed. 3-Methyl-1H-pyrazolones were synthesized from substituted hydrazides using various solid supports under microwave irradiation (MWI). The results obtained highlight the versatility of the solid supports. All synthesized compounds were screened for their antibacterial activity against gram positive and gram negative bacteria and showed good to significant activity, as well as demonstrated significant antifungal activity against Candida albicans ATCC 10231 and C. krusei GO3.
\end{abstract}

Keywords: Niementowski reaction, microwave, pyrazolone, quinazolinone

\section{Introduction}

The present day industrialization has led to immense environmental deterioration. The increasing environmental consciousness throughout the world has put a pressing need to develop an alternate synthetic approach for biologically and synthetically important compounds. This requires a new approach, which will reduce the material and energy intensity of chemical processes and products, minimize or eliminate the dispersion of harmful chemicals in the environment in a way that enhances the industrially benign approach and meets the challenges of

green chemisty. ${ }^{1}$ One of the advances in this area where substantial progress has been made is the microwave-assisted solid-support synthesis. ${ }^{2}$ Aluminas can be selected as acidic or basic or neutral catalyst depending on the type of organic reaction. The dream of green chemistry has 
thus come true with the help of the "neat reaction" technology. "Neat reaction" is an alternative solvent-free approach in which a mixture of reactants in the absence of solvent is irradiated under microwaves. The striking features are improved yield, shorter reaction time and easier work-up. This justifies the definition of "no solvent" because it completely eliminates the need of a solvent. However, absorption of the conventional heating procedures in the absence of solvent may lead to charring.

Quinazolinone derivatives attract a widespread interest due to the diverse biological activities $^{3}$ associated with them. They are pharmaceutically important as antituberculars ${ }^{4}$, antibacterial $^{5}$, antiparkinsons ${ }^{6}$, antihelmintics ${ }^{6}$, and they also show blood platelet antiaggregating activity. ${ }^{7}$ Formation of 2 -alkyl-4(3H)-quinazolinones by condensation of anthranilic acid or substituted anthranilic acid and amides is designated as the Niementowski reaction. ${ }^{8}$ Pyrazolones are associated with broad spectrum of biological activities including antifungal, antibacterial, anti-inflammatory properties. ${ }^{9-11}$ Hydrazide derivatives have been extensively used as a good precursor for the synthesis of these derivatives. ${ }^{12-13}$

Keeping in view the biological importance of the above mentioned heterocyclic compounds and in continuation to our endeavor towards environmentally benign synthesis, ${ }^{14}$ we report herein the synthesis of 6-substituted-2-(methyl/phenyl)-3-[-2-(methyl-5-oxo-2,5-dihydro-1Hpyrazol-1-yl)-2-oxo(ethyl/phenyl)]-quinazolin-4(3H)-ones 3(a-l) from 6-substitued-2(methyl/phenyl)-4-oxoquinazolin-3(4H)-yl)-(aceto/benzo) hydrazide 2(a-l) using different solid supports under MWI as well as with the conventional techniques (Table 1). These derivatives were screened for their antifungal and antibacterial activity against Candida albicans and E.coli as well as S. aureus respectively by the cup-plate method $^{15}$ (Table 2).

Table 1. Observed Yield and Reaction time of compounds 3 (a-1)

\begin{tabular}{|c|c|c|c|c|c|c|c|c|c|c|}
\hline \multirow{2}{*}{$\begin{array}{l}\text { Comp. } \\
\text { No. }\end{array}$} & \multirow[t]{2}{*}{$\mathrm{Y}$} & \multirow[t]{2}{*}{$\mathrm{R}$} & \multirow[t]{2}{*}{$X$} & \multirow[t]{2}{*}{$\mathrm{mp} /{ }^{\circ} \mathrm{C}$} & \multicolumn{4}{|c|}{$\begin{array}{c}\text { Microwave Irradiation } \\
\text { (neat Conditions) }^{(a)}\end{array}$} & \multicolumn{2}{|c|}{ Yield $\%{ }^{(b)}$} \\
\hline & & & & & $t_{1} / \min$ & $p_{1} / w$ & $t_{2} / \min$ & $p_{2} / w$ & A & B \\
\hline $3 a$ & $\mathrm{Br}$ & $\mathrm{CH}_{3}$ & $4-\mathrm{C}_{6} \mathrm{H}_{4}$ & $175-178$ & 5.0 & 400 & 4.0 & 500 & 26 & 90 \\
\hline $3 b$ & $\mathrm{H}$ & $\mathrm{CH}_{3}$ & $4-\mathrm{C}_{6} \mathrm{H}_{4}$ & $182-185$ & 4.0 & 320 & 5.0 & 450 & 80 & 92 \\
\hline $3 c$ & $\mathrm{Br}$ & $\mathrm{C}_{6} \mathrm{H}_{5}$ & $4-\mathrm{C}_{6} \mathrm{H}_{4}$ & $252-256$ & 5.0 & 450 & 3.5 & 450 & 72 & 85 \\
\hline $3 d$ & $\mathrm{H}$ & $\mathrm{C}_{6} \mathrm{H}_{5}$ & $4-\mathrm{C}_{6} \mathrm{H}_{4}$ & 196-198 & 4.0 & 400 & 5.0 & 400 & 78 & 90 \\
\hline $3 e$ & $\mathrm{Br}$ & $\mathrm{CH}_{3}$ & $\mathrm{CH}_{2}$ & $225-229$ & 5.0 & 450 & 4.0 & 500 & 80 & 92 \\
\hline $3 f$ & $\mathrm{H}$ & $\mathrm{CH}_{3}$ & $\mathrm{CH}_{2}$ & $187-191$ & 5.0 & 320 & 4.0 & 450 & 78 & 90 \\
\hline $3 g$ & $\mathrm{Br}$ & $\mathrm{C}_{6} \mathrm{H}_{5}$ & $\mathrm{CH}_{2}$ & $180-182$ & 4.5 & 400 & 3.5 & 500 & 82 & 95 \\
\hline $3 \mathrm{~h}$ & $\mathrm{H}$ & $\mathrm{C}_{6} \mathrm{H}_{5}$ & $\mathrm{CH}_{2}$ & $174-177$ & 5.0 & 320 & 4.5 & 450 & 74 & 88 \\
\hline $3 \mathrm{i}$ & $\mathrm{Br}$ & $\mathrm{CH}_{3}$ & $3-\mathrm{C}_{6} \mathrm{H}_{4}$ & $126-128$ & 4.0 & 450 & 4.0 & 500 & 72 & 85 \\
\hline $3 \mathrm{j}$ & $\mathrm{H}$ & $\mathrm{CH}_{3}$ & $3-\mathrm{C}_{6} \mathrm{H}_{4}$ & $195-197$ & 4.0 & 420 & 5.0 & 350 & 70 & 89 \\
\hline
\end{tabular}


Table 1. Continued

\begin{tabular}{lllllllllll}
\hline $3 \mathrm{k}$ & $\mathrm{Br}$ & $\mathrm{C}_{6} \mathrm{H}_{5}$ & $3-\mathrm{C}_{6} \mathrm{H}_{4}$ & $275-278$ & 4.5 & 400 & 3.5 & 450 & 77 & 90 \\
31 & $\mathrm{H}$ & $\mathrm{C}_{6} \mathrm{H}_{5}$ & $3-\mathrm{C}_{6} \mathrm{H}_{4}$ & $248-250$ & 5.0 & 400 & 4.0 & 350 & 75 & 92 \\
\hline
\end{tabular}

(a) Microwave irradiations were carried out in a Kenstar microwave oven, model No. OM9925E (2560 MHz, 750 Watts). ${ }^{\text {(b) }}$ A: Conventional heating; B: Microwave heating.

Table 2. Antibacterial and Antifungal activity screening data of compounds 3(a-1)

\begin{tabular}{|c|c|c|c|c|c|c|}
\hline \multirow{3}{*}{$\begin{array}{c}\text { Compound } \\
\text { No. }\end{array}$} & \multicolumn{4}{|c|}{ Antibacterial in $(\mu \mathrm{g} / \mathrm{ml})$} & \multicolumn{2}{|c|}{ Antifungal in $(\mu \mathrm{g} / \mathrm{ml})$} \\
\hline & \multicolumn{2}{|c|}{ Gram positive } & \multicolumn{2}{|c|}{ Gram negative } & \multirow{2}{*}{$\frac{\text { Candida }}{\text { ATCC } 10231}$} & \multirow{2}{*}{$\frac{\text { C.krusei }}{\text { G03 }}$} \\
\hline & S. $\mathrm{a}^{\mathrm{a}}$ & B.s ${ }^{b}$ & E.c $c^{c}$ & P. $a^{d}$ & & \\
\hline $3 a$ & 12 & 14 & 15 & 14 & 11 & 10 \\
\hline $3 b$ & 11 & 10 & 13 & 12 & $21 \mathrm{~h}$ & 22 \\
\hline $3 c$ & 16 & 17 & 11 & 10 & 20 & $18 \mathrm{~h}$ \\
\hline $3 d$ & 08 & 09 & 09 & 07 & 08 & 05 \\
\hline $3 \mathrm{e}$ & 13 & 12 & 11 & 10 & 20 & 22 \\
\hline $3 f$ & 06 & 08 & 04 & 09 & $18 \mathrm{~h}$ & 24 \\
\hline $3 g$ & 11 & 09 & 17 & 16 & 07 & 09 \\
\hline $3 \mathrm{~h}$ & 09 & 08 & 18 & 16 & 10 & 11 \\
\hline $3 \mathrm{i}$ & 16 & 14 & 11 & 10 & 17 & $20 \mathrm{~h}$ \\
\hline $3 \mathrm{j}$ & 05 & 06 & 06 & 07 & 05 & 08 \\
\hline $3 \mathrm{k}$ & 07 & 09 & 07 & 06 & 07 & 09 \\
\hline 31 & 17 & 19 & 08 & 11 & $15 \mathrm{~h}$ & 18 \\
\hline \multicolumn{7}{|c|}{ Zone of inhibition of standard drugs $(\mu \mathrm{g} / \mathrm{ml})$} \\
\hline Ampicillin & 17 & 19 & 18 & 20 & - & - \\
\hline Amoxicillin & 19 & 22 & 21 & 23 & - & - \\
\hline Penicillin & 20 & 21 & 18 & 17 & - & - \\
\hline Flucanozole & - & - & - & - & 25 & 28 \\
\hline
\end{tabular}

Where ${ }^{\text {a }}$ S.aureus. ${ }^{b}$ B.subtilis. ${ }^{c}$ E.coli. ${ }^{d}$ P.aeruginosa ' $h$ ' denotes hazy zone.

\section{Results and Discussion}

The amides or amidines generally used in the Niementowski reaction were replaced by formic acid and primary aromatic or heteroaromatic amines, which afforded 3-substituted-4(3H) quinazolinones instead of the corresponding 2-substituted derivative. In continuation of our earlier work on microwave synthesis ${ }^{16}$ and with respect to the advantage of coupling, solvent free conditions ${ }^{17}$ with microwave irradiation (MWI), equimolar amounts of neat reactants were 
mixed and irradiated under microwaves. This proved to be a high-yielding protocol (Table 1). Inspired by the high yields obtained using formic acid, a further study of $4(3 \mathrm{H})$ quinazolinones synthesis, as a possible Niementowski reaction, was carried out using different aromatic and heteroaromatic carboxylic acid, amine and carboxylic acid were mixed and irradiated under microwaves to yield 2,3-disubstituted-4(3H) quinazolinones 1(a-l). Good yields were obtained in less irradiation time (Table 1 ) compared to the conventional procedure ${ }^{17}$ requiring 5 -substituted anthranilic acid, acetyl chloride, pyridine and amino acids and long refluxing time. The hydrazide 2(a-l) from the corresponding acid was prepared by hydrazine hydrate. Novel route towards synthesis of 6-bromo-2-methyl-3-[-4-[3-methyl-5-oxo-2,5-dihydro-1H-pyrazol-1yl)carbonyl]phenyl \} quinazoline-4(3H)-one 3(a) from 4-(6-bromo-2-methyl-4-oxoquinazolin3(4H)-yl) benzohydrazide 2(a) using different solid supports as neutral alumina and by conventional technique using ethyl acetoacetate in ethanol solvent. It gave new 3-methyl-1H 5pyrazolone in good yield.

The structures of 3(a-l) were confirmed on the basis of spectral and analytical data. IR spectra showed disappearance of bands at $1710-1720 \mathrm{~cm}^{-1}$ to $\mathrm{C}=\mathrm{O}$ of carboxylic group and 3400$3300 \mathrm{~cm}^{-1}$ due to NH of amine and appearance of the band at $1685-1699 \mathrm{~cm}^{-1}(\mathrm{C}=\mathrm{O})$ and 1590 $1615 \mathrm{~cm}^{-1}(\mathrm{C}=\mathrm{N})$. In ${ }^{1} \mathrm{H} \mathrm{NMR}$, appearance of the signal at $\delta 10.5-12 \mathrm{ppm}$ due to carboxylic proton of amino carboxylic acid and a broad signal at $\delta 3-4 \mathrm{ppm}$ due to amine confirmed the formation of products 3(a-l). The final structures were established by the appearance of band at $1595 \mathrm{~cm}^{-1}$ and disappearance of band at $1730 \mathrm{~cm}^{-1}$ of carboxylic acid. The disappearance of band at $1720 \mathrm{~cm}^{-1}(\mathrm{C}=\mathrm{O})$ of ester and appearance of band at $1660 \mathrm{~cm}^{-1}$ due to the pyrazolone ring. ${ }^{1} \mathrm{H}$ NMR signal at $\delta 5.5$ due to $\mathrm{H}-4$ proton and at $\delta 2.3$ due to the methyl group protons was present. Elemental analysis is furnished in the experimental section. The reaction pathways are depicted in Scheme 1. 
<smiles>[Y]c1ccc2nc([R])n([X])c(=O)c2c1</smiles><smiles>[Y]c1ccc(N)c(C(=O)O)c1</smiles><smiles>[R]c1ccc2nc([R])n([X]C(=O)OCC)c(=O)c2c1</smiles>

$1(a-l)$<smiles>[Y]c1ccc2nc([R])n([X]C(=O)n3[nH]c(C)cc3=O)c(=O)c2c1</smiles>

\section{Scheme 1}<smiles>[Y]c1ccc2nc([R])n([X]c3ccc(C(N)=O)cc3)c(=O)c2c1</smiles>

$2(a-I)$

Where: C Conventional Method: - Pyridine, Reflux 6-8 hrs.

M Microwave Method: - $\mu \nu\left(\mathrm{t}_{1}=5 \mathrm{~min}\right.$ and $\left.\mathrm{t}_{2}=4 \mathrm{~min}\right)$ for $\left(\mathrm{p}_{1}=400 \mathrm{w}\right.$ and $\left.\mathrm{p}_{2}=500 \mathrm{w}\right)$.

B $\mathrm{SOCl}_{2}$, reflux 1-2 hrs, $\mathrm{C}_{2} \mathrm{H}_{5} \mathrm{OH}$ reflux 5-6 hrs.

D Hydrazine hydrate $99 \%$, reflux $6 \mathrm{hrs}$ in ethanol as solvent.

C' Reflux 6-8 hrs, $\mathrm{CH}_{3} \mathrm{COCH}_{2} \mathrm{COOC}_{2} \mathrm{H}_{5}$ in ethanol as solvent.

M' Microwave Method: - MWI 5-7 min, Neutral Alumina $\mathrm{CH}_{3} \mathrm{COCH}_{2} \mathrm{COOC}_{2} \mathrm{H}_{5}$ in ethanol as solvent and dried in air.

\begin{tabular}{cccccccc}
\hline CodeNo. & $\mathrm{Y}$ & $\mathrm{R}$ & $\mathrm{X}$ & CodeNo. & $\mathrm{Y}$ & $\mathrm{R}$ & $\mathrm{X}$ \\
\hline $3 \mathrm{a}$ & $\mathrm{Br}$ & $\mathrm{CH}_{3}$ & $4-\mathrm{C}_{6} \mathrm{H}_{4}$ & $3 \mathrm{~g}$ & $\mathrm{Br}$ & $\mathrm{C}_{6} \mathrm{H}_{5}$ & $\mathrm{CH}_{2}$ \\
$3 \mathrm{~b}$ & $\mathrm{H}$ & $\mathrm{CH}_{3}$ & $4-\mathrm{C}_{6} \mathrm{H}_{4}$ & $3 \mathrm{~h}$ & $\mathrm{H}$ & $\mathrm{C}_{6} \mathrm{H}_{5}$ & $\mathrm{CH}_{2}$ \\
$3 \mathrm{c}$ & $\mathrm{Br}$ & $\mathrm{C}_{6} \mathrm{H}_{5}$ & $4-\mathrm{C}_{6} \mathrm{H}_{4}$ & $3 \mathrm{i}$ & $\mathrm{Br}$ & $\mathrm{CH}_{3}$ & $3-\mathrm{C}_{6} \mathrm{H}_{4}$ \\
$3 \mathrm{~d}$ & $\mathrm{H}$ & $\mathrm{C}_{6} \mathrm{H}_{5}$ & $4-\mathrm{C}_{6} \mathrm{H}_{4}$ & $3 \mathrm{j}$ & $\mathrm{H}$ & $\mathrm{CH}_{3}$ & $3-\mathrm{C}_{6} \mathrm{H}_{4}$ \\
$3 \mathrm{e}$ & $\mathrm{Br}$ & $\mathrm{CH}_{3}$ & $\mathrm{CH}_{2}$ & $3 \mathrm{k}$ & $\mathrm{Br}$ & $\mathrm{C}_{6} \mathrm{H}_{5}$ & $3-\mathrm{C}_{6} \mathrm{H}_{4}$ \\
$3 \mathrm{f}$ & $\mathrm{H}$ & $\mathrm{CH}_{3}$ & $\mathrm{CH}_{2}$ & 31 & $\mathrm{H}$ & $\mathrm{C}_{6} \mathrm{H}_{5}$ & $3-\mathrm{C}_{6} \mathrm{H}_{4}$ \\
\hline
\end{tabular}




\section{Experimental Section}

General Procedures. All microwave reactions were carried out in a Kenstar microwave oven, model no. OM9925E (2560 MHz, 750 Watts). Melting points were determined in PMP-DM scientific melting point apparatus and were uncorrected. The IR spectra were recorded in $\mathrm{KBr}$ on a "Perkin-Elmer RX 1 FT-IR" spectrophotometer (serial No. 51448) and ${ }^{1} \mathrm{H}$ NMR spectra were recorded on a Bruker A/C $300 \mathrm{~F}$ spectrophotometer and chemical shifts $(\delta)$ are in ppm relative to internal tetramethylsilane. The mass spectra were run on a Varian model MAT MS-311 spectrometer at $70 \mathrm{eV}$. Purity of the compounds in addition to elemental analysis was checked by TLC.

\section{General synthesis of 6-Bromo-2-methyl-4-oxoquinazolin-3(4H)-yl) benzoic acid 1(a)}

\section{(a) By conventional heating}

In a $250 \mathrm{ml}$ beaker, 5-bromoanthranilic acid $(2.16 \mathrm{~g}, 0.01 \mathrm{~mol})$ in presence of pyridine $(0.79 \mathrm{~g}$, $0.01 \mathrm{~mol})$ was taken. Acetyl chloride $(0.78 \mathrm{~g}, 0.01 \mathrm{~mol})$ at $0-5{ }^{\circ} \mathrm{C}$ was added dropwise to afford cyclization to give 2-methyl-6-bromo-4H-3,1-benzoxazin-4-one. The 6-bromobenzoxazone and p-aminobenzoic acid $(1.37 \mathrm{~g}, 0.01 \mathrm{~mol})$ were dissolved in pyridine and refluxed for $6 \mathrm{hrs}$. Excess of solvent was distilled off. The resulting mixture was cooled and poured over crushed ice. The solid was filtered, washed with cold water, and recrystallised from glacial acetic acid. The \% yield is $75 \%$, m.p $175^{\circ} \mathrm{C}$. Similarly 6-bromo-2-phenyl-3-[4-(carboxyphenyl)]-4-(3H)quinazolinones were also synthesized by using benzoyl chloride in place of acetyl chloride. The different 6-substituted compounds have been synthesized by using various amino carboxylic acids by above method.

\section{(b) By microwave heating}

Equimolar amounts of 6-bromo-anthranilic acid $(2.16 \mathrm{~g}, 0.01 \mathrm{~mol})$, p-amino benzoic acid $(1.37 \mathrm{~g}, 0.01 \mathrm{~mol})$, and acetic acid $(0.60 \mathrm{~g}, 0.01 \mathrm{~mol})$ were put in an Erlenmeyer flask and irradiated under microwaves in two stages $\left(t_{1}=5 \mathrm{~min}\right.$ and $\left.t_{2}=4 \mathrm{~min}\right)$ at two different power levels $\left(\mathrm{p}_{1}=400 \mathrm{w}\right.$ and $\mathrm{p}_{2}=500 \mathrm{w}$ ) (Table 1 ) respectively. Reactions progress was monitored by TLC. Upon completion of reaction, the reaction mixture was worked up with an excess of 10\% $\mathrm{NaHCO}_{3}$ solution and recrystallised from ethanol. The \% yield is $90 \%$, m.p $175^{\circ} \mathrm{C}$. Similarly 6bromo-2-phenyl-4-oxoquinazolin-3(4H)-yl) benzoic acid 1(b) was also synthesized by using benzoic acid in place of acetic acid. The different 6-substituted compounds have been synthesized by using various amino carboxylic acids by above method.

6-Bromo-2-methyl-4-oxoquinazolin-3(4H)-yl) benzoic acid. Reagents: For A 6-bromo anthranilic acid $(216 \mathrm{mg})$, pyridine $(79 \mathrm{mg})$, acetyl chloride $(78 \mathrm{mg})$, p-amino benzoic acid (137 mg). Yield: 75\%; For B 6-bromo anthranilic acid (216 mg), p-amino benzoic acid (137 mg), acetic acid $(60 \mathrm{mg})$. Yield: 90\%; mp 175 $\mathrm{C}$. IR $(\mathrm{KBr}): 1699(\mathrm{C}=\mathrm{O}), 1600(\mathrm{C}=\mathrm{N})$ quinazoline; 1680, $1685(\mathrm{C}=\mathrm{O}), 3060$ (aromatic C-H), $1310(\mathrm{~N}-\mathrm{C}-\mathrm{N}), 2890(\mathrm{COOH}), 565$ (C-Br). ${ }^{1} \mathrm{H}-\mathrm{NMR}$ : 
1.2 (s, 3H, Me), 7.1-8.1 (m, 7H, Ph \& H-5, H-7, H-8), 9.5-10.2 (s, 1H, COOH). MS: m/z 359 $[\mathrm{M}]^{+}$. Anal calcd. for $\mathrm{C}_{16} \mathrm{H}_{11} \mathrm{BrN}_{2} \mathrm{O}_{3}$ : C, 53.50; H, 3.04; N, 7.80; Found: C, 53.52; H, 3.01; N, 7.78 .

\section{Synthesis of 4-(6-bromo-2-methyl-4-oxoquinazolin-3(4H)-yl) benzoyl chloride} (a) By conventional heating

In a $250 \mathrm{ml}$ round bottom flask 6-bromo-2-methyl-4-oxoquinazolin-3(4H)-yl) benzoic acid 1(a) $(3.59 \mathrm{~g}, 0.01 \mathrm{~mol})$ and thionyl chloride $(1.785 \mathrm{~g}, 0.015 \mathrm{~mol})$ were added and refluxed for $1-2 \mathrm{hrs}$. Excess of thionyl chloride was then distilled off and then the reaction mixture was cooled in an ice bath. The solid was separated and immediately used for the next reaction.

\section{Synthesis of 4-(6-bromo-2-methyl-4-oxoquinazolin-3(4H)-yl) benzohydrazide 2(a)}

(a) By conventional heating

In a $250 \mathrm{ml}$ round bottom flask 4-(6-bromo-2-methyl-4-oxoquinazolin-3(4H)-yl) benzoyl chloride $(3.775 \mathrm{~g}, 0.01 \mathrm{~mol})$ in ethanol $(40 \mathrm{ml})$ was taken, hydrazine hydrate $(95 \% 1.00 \mathrm{~g}$, $0.02 \mathrm{~mol}$ ) was added slowly with constant stirring and the reaction mixture was kept under reflux condition for $6 \mathrm{hrs}$. The solvent was then removed by distillation under reduced pressure and the residue was poured into ice-cold water, and recrystallised by using ethanol.

4-(6-Bromo-2-methyl-4-oxoquinazolin-3(4H)-yl) benzohydrazide. Yield: $76 \%$; $\mathrm{mp} 180^{\circ} \mathrm{C}$. IR $(\mathrm{KBr}): 3410\left(\mathrm{NH}_{2}\right), 3280(\mathrm{NH}), 1695(\mathrm{C}=\mathrm{O}), 1599(\mathrm{C}=\mathrm{N})$ quinazoline; 1675, $1690(\mathrm{C}=\mathrm{O}), 3040$ (aromatic C-H), 1530 (C---C of aromatic ring), 1312 (N-C-N), 568 (C-Br). ${ }^{1} \mathrm{H}-\mathrm{NMR}$ $\left(\mathrm{CDCl}_{3}+\mathrm{DMSO}_{-} \mathrm{d}_{6}\right): 6.1\left(\mathrm{t}, 1 \mathrm{H},-\mathrm{NHNH}_{2}\right.$, exchangeable with $\left.\mathrm{D}_{2} \mathrm{O}\right), 5.96\left(\mathrm{~d}, 2 \mathrm{H}, \mathrm{NHNH}_{2}\right.$, exchangeable with $\mathrm{D}_{2} \mathrm{O} 1.4$ (s, 3H, Me), 7.2-8.15 (m, 7H, Ph \& H-5, H-7, H-8). MS: m/z 373 $[\mathrm{M}]^{+}$. Anal. calcd. for $\mathrm{C}_{16} \mathrm{H}_{11} \mathrm{BrN}_{2} \mathrm{O}_{3}: \mathrm{C}, 55.02 ; \mathrm{H}, 3.04 ; \mathrm{N}, 16.88$; Found: $\mathrm{C}, 55.04 ; \mathrm{H}, 3.01 ; \mathrm{N}$, 16.92 .

General synthesis of 6-bromo-2-methyl-3-\{4-[(3-methyl-5-oxo-2,5-dihydro-1H-pyrazol-1yl)carbonyl] phenyl\}quinazolin-4(3H)-one 3(a)

\section{(a) By conventional heating}

In a round bottom flask a solution of 4-(6-bromo-2-methyl-4-oxoquinazolin-3(4H)-yl) benzohydrazide 2(a) $(3.732 \mathrm{~g}, 0.01 \mathrm{~mol})$ in ethanol $(30 \mathrm{ml})$ was taken. Ethyl acetoacetate $(1.30 \mathrm{ml}$, $0.01 \mathrm{~mol}$ ) was added and it was refluxed on a water bath for $6 \mathrm{hrs}$. Excess of solvent was then distilled off. The resulting solid was crystallized from ethanol to give above product.

\section{B) By microwave heating}

To the ethanolic solution $(20 \mathrm{ml})$ of $2 \mathrm{a}(0.01 \mathrm{~mol})$ and ethyl acetoacetate $(1.30 \mathrm{ml}, 0.01 \mathrm{~mol})$ in $100 \mathrm{ml}$ beaker, neutral alumina (20 g) was added. The reaction mixture was stirred well and dried in air. It was placed in an alumina bath and subjected to MWI intermittently at an interval of $30 \mathrm{~s}$ for specified time (Table I). On completion of the reaction, as monitored by TLC examination (at an interval of 30s), the product was extracted with ethanol $(3 \times 10 \mathrm{~mL})$. Removal 
of solvent under reduced pressure gave the desired product (Table 2) which was recrystallised from ethanol.

6-Bromo-2-methyl-3-\{4-[(3-methyl-5-oxo-2,5-dihydro-1H-pyrazol-1-yl)carbonyl]phenyl\} quinazolin-4(3H)-one (3a). For A Yield: 75\%; For B Yield: 90\% mp 219-220 ${ }^{\circ} \mathrm{C}$. IR (KBr): $1699(\mathrm{C}=\mathrm{O}), 1600(\mathrm{C}=\mathrm{N})$ Quinazoline; 1680, 1685, $(\mathrm{C}=\mathrm{O}), 1310\left(\mathrm{C}-\mathrm{CH}_{3}\right)$ Pyrazolone, 3060 (aromatic C-H), 1529 (C---C of aromatic ring) 1317 (N-C-N), 547 (C-Br). ${ }^{1} \mathrm{H}-\mathrm{NMR}$ ( $\left.\mathrm{CDCl}_{3}+\mathrm{DMSO}-\mathrm{d6}\right): 1.2$ (s, 3H, Me), 7.1-8.1 (m, 7H, Ph \& H-5, H-7, H-8), 2.3 (s, 3H, Me), 5.5 (s, 1H, CH), 8.4 (br, $1 \mathrm{H}, \mathrm{NH})$ Pyrazolone. MS: $\mathrm{m} / \mathrm{z}[439]^{+}$. Anal. calcd. for $\mathrm{C}_{20} \mathrm{H}_{15} \mathrm{BrN}_{4} \mathrm{O}_{3}$ : C, 54.69; H, 3.44; N, 12.75; Found: C, 54.67; H, 3.42; N, 12.77.

2-Methyl-3-\{4-[(3-methyl-5-oxo-4,5-dihydro-1H-pyrazol-1-yl)carbonyl]phenyl\}quinazolin4(3H)-one (3b). For A Yield: 80\%; For B Yield: 92\% mp 182-185 ${ }^{\circ} \mathrm{C}$. IR (KBr): $1678(\mathrm{C}=\mathrm{O})$, $1605(\mathrm{C}=\mathrm{N})$ Quinazoline; 1698, 1710, $(\mathrm{C}=\mathrm{O}), 1308\left(\mathrm{C}-\mathrm{CH}_{3}\right)$ Pyrazolone, 3065 (aromatic $\left.\mathrm{C}-\mathrm{H}\right)$, 1521 (C---C of aromatic ring) $1318(\mathrm{~N}-\mathrm{C}-\mathrm{N}),{ }^{1} \mathrm{H}-\mathrm{NMR}(\mathrm{DMSO}-\mathrm{d} 6): 1.3$ (s, 3H, Me), 7.3-8.4 (m, $8 \mathrm{H}$, Ph \& H-5 to H-8), 2.4 (s, 3H, Me), 5.7 (s, 1H, CH), 8.3 (br, 1H, NH) Pyrazolone. MS: m/z $[360]^{+}$. Anal. calcd. for $\mathrm{C}_{20} \mathrm{H}_{16} \mathrm{~N}_{4} \mathrm{O}_{3}$ : C, 66.66; H, 4.48; N, 15.55; Found: $\mathrm{C}, 66.68 ; \mathrm{H}, 4.46 ; \mathrm{N}$, 15.56 .

6-Bromo-3-\{4-[(3-methyl-5-oxo-4,5-dihydro-1H-pyrazol-1-yl)carbonyl]phenyl\}-2-phenyl quinazolin-4(3H)-one (3c). For A Yield: 72\%; For B Yield: 85\% mp 252 ${ }^{\circ} \mathrm{C}$. IR (KBr): 1672 $(\mathrm{C}=\mathrm{O}), 1611(\mathrm{C}=\mathrm{N})$ Quinazoline; 1685, 1692, $(\mathrm{C}=\mathrm{O}), 1312\left(\mathrm{C}-\mathrm{CH}_{3}\right)$ Pyrazolone, 3045 (aromatic $\mathrm{C}-\mathrm{H}), 1538$ (C---C of aromatic ring) 1320 (N-C-N), 560 (C-Br). ${ }^{1} \mathrm{H}-\mathrm{NMR}$ (DMSO-d6): 7.3-8.4 (m, 12H, 2 x Ph \& H-5, H-7, H-8), 2.4 (s, 3H, Me), 5.6 (s, 1H, CH), 8.4 (br, 1H, NH) Pyrazolone. MS: $\mathrm{m} / \mathrm{z}[501]^{+}$. Anal. calcd. for $\mathrm{C}_{25} \mathrm{H}_{17} \mathrm{BrN}_{4} \mathrm{O}_{3}: \mathrm{C}, 59.89 ; \mathrm{H}, 3.42 ; \mathrm{N}, 11.18$; Found: C, 59.91; H, 3.41; N, 11.16.

3-\{4-[(3-Methyl-5-oxo-4,5-dihydro-1H-pyrazol-1-yl)carbonyl] phenyl\}-2-phenyl quinazolin4(3H)-one (3d). For A Yield: 78\%; For B Yield: 90\% mp 196-198 ${ }^{\circ}$. IR (KBr): $1685(\mathrm{C}=\mathrm{O})$, $1598(\mathrm{C}=\mathrm{N})$ Quinazoline; 1705, 1715, $(\mathrm{C}=\mathrm{O}), 1307\left(\mathrm{C}-\mathrm{CH}_{3}\right)$ Pyrazolone, 3049 (aromatic $\left.\mathrm{C}-\mathrm{H}\right)$, $1530\left(\mathrm{C}---\mathrm{C}\right.$ of aromatic ring) $1311(\mathrm{~N}-\mathrm{C}-\mathrm{N}) .{ }^{1} \mathrm{H}-\mathrm{NMR}\left(\mathrm{CDCl}_{3}+\mathrm{DMSO}-\mathrm{d} 6\right)$ : 7.2-8.0 (m, 13H, 2 $\mathrm{x} \mathrm{Ph} \& \mathrm{H}-5$ to H-8), 2.4 (s, 3H, Me), 5.4 (s, 1H, CH), 8.7 (br, 1H, NH) Pyrazolone. MS: m/z [422] $]^{+}$Anal. calcd. for $\mathrm{C}_{25} \mathrm{H}_{18} \mathrm{~N}_{4} \mathrm{O}_{3}$ : C, 71.08; H, 4.29; N, 13.26; Found: C, 71.10; H, 4.27; N, 13.29 .

6-Bromo-2-methyl-3-[2-(3-methyl-5-oxo-4,5-dihydro-1H-pyrazol-1-yl)-2-oxoethyl] quinazolin-4(3H)-one (3e). For A Yield: 80\%; For B Yield: 92\% mp 225 C. IR (KBr): 1695 $(\mathrm{C}=\mathrm{O}), 1590(\mathrm{C}=\mathrm{N})$ Quinazoline; 1686, 1699, $(\mathrm{C}=\mathrm{O}), 1315\left(\mathrm{C}-\mathrm{CH}_{3}\right)$ Pyrazolone, 3055 (aromatic $\mathrm{C}-\mathrm{H}), 1542$ (C---C of aromatic ring) 1310 (N-C-N), 552 (C-Br). ${ }^{1} \mathrm{H}-\mathrm{NMR}$ (DMSO-d6): 1.2 (s, $3 \mathrm{H}, \mathrm{Me}), 7.1-8.5$ (m, 3H, H-5, H-7, H-8), 1.1 (s, 2H, $\left.\mathrm{CH}_{2}\right) 2.3$ (s, 3H, Me), 5.5 (s, 1H, CH), 8.4 (br, 1H, NH) Pyrazolone. MS: m/z [455] . Anal. calcd. for $\mathrm{C}_{21} \mathrm{H}_{19} \mathrm{BrN}_{4} \mathrm{O}_{3}: \mathrm{C}, 55.40 ; \mathrm{H}, 4.21 ; \mathrm{N}$, 12.31; Found: C, 55.42; H, 4.23; N, 12.29.

2-Methyl-3-[2-(3-methyl-5-oxo-4,5-dihydro-1H-pyrazol-1-yl)-2-oxoethyl]quinazoline-4(3H)one (3f). For A Yield: 78\%; For B Yield: 90\% mp 187 C. IR (KBr): $1705(\mathrm{C}=\mathrm{O}), 1605(\mathrm{C}=\mathrm{N})$ 
Quinazoline; 1682, 1685, $(\mathrm{C}=\mathrm{O}), 1307\left(\mathrm{C}-\mathrm{CH}_{3}\right)$ Pyrazolone, 3080 (aromatic $\left.\mathrm{C}-\mathrm{H}\right), 1540$ (C---C of aromatic ring) $1321(\mathrm{~N}-\mathrm{C}-\mathrm{N}) .{ }^{1} \mathrm{H}-\mathrm{NMR}\left(\mathrm{CDCl}_{3}\right): 1.2$ (s, 3H, Me), 7.3-7.9 (m, 4H, H-5 to H-8), $1.2\left(\mathrm{~s}, 2 \mathrm{H}, \mathrm{CH}_{2}\right) 2.5$ (s, 3H, Me), $5.3(\mathrm{~s}, 1 \mathrm{H}, \mathrm{CH}), 8.2$ (br, 1H, NH) Pyrazolone. MS: m/z [376] $]^{+}$ Anal. calcd. for $\mathrm{C}_{20} \mathrm{H}_{20} \mathrm{~N}_{4} \mathrm{O}_{3}$ : C, 67.01; H, 5.36; N, 14.88; Found: C, 67.04; H, 5.34; N, 14.85 .

6-Bromo-3-[2-(3-methyl-5-oxo-4,5-dihydro-1H-pyrazol-1-yl)-2-oxoethyl]-2-phenyl quinazolin-4(3H)-one (3g). For A Yield: 82\%; For B Yield: $95 \% \mathrm{mp} 180^{\circ} \mathrm{C}$. IR (KBr): 1699 $(\mathrm{C}=\mathrm{O}), 1597(\mathrm{C}=\mathrm{N})$ Quinazoline; 1710, 1686, $(\mathrm{C}=\mathrm{O}), 1318\left(\mathrm{C}-\mathrm{CH}_{3}\right)$ Pyrazolone, 3045 (aromatic $\mathrm{C}-\mathrm{H}), 1538$ (C---C of aromatic ring) $1325(\mathrm{~N}-\mathrm{C}-\mathrm{N}), 560(\mathrm{C}-\mathrm{Br}) .{ }^{1} \mathrm{H}-\mathrm{NMR}\left(\mathrm{CDCl}_{3}\right)$ : 7.2-7.8 (m, 8H, Ph, H-5, H-7, H-8), 1.3 (s, 2H, $\left.\mathrm{CH}_{2}\right) 2.3$ (s, 3H, Me), 5.5 (s, 1H, CH), 8.4 (br, 1H, NH) Pyrazolone. MS: $\mathrm{m} / \mathrm{z}$ [439] $]^{+}$. Anal. calcd. for $\mathrm{C}_{20} \mathrm{H}_{15} \mathrm{BrN}_{4} \mathrm{O}_{3}: \mathrm{C}, 54.59 ; \mathrm{H}, 3.40 ; \mathrm{N}, 12.85$; Found: C, 54.62; H, 3.38; N, 12.84 .

3-[2-(3-Methyl-5-oxo-4,5-dihydro-1H-pyrazol-1-yl)-2-oxoethyl]-2-phenylquinazolin-4(3H)one (3h). For A Yield: 74\%; For B Yield: 88\% mp 174 C. IR (KBr): $1685(\mathrm{C}=\mathrm{O}), 1599(\mathrm{C}=\mathrm{N})$ Quinazoline; 1698, 1705, $(\mathrm{C}=\mathrm{O}), 1310\left(\mathrm{C}-\mathrm{CH}_{3}\right)$ Pyrazolone, 3058 (aromatic $\left.\mathrm{C}-\mathrm{H}\right), 1535(\mathrm{C}---\mathrm{C}$ of aromatic ring) $1320(\mathrm{~N}-\mathrm{C}-\mathrm{N}) .{ }^{1} \mathrm{H}-\mathrm{NMR}\left(\mathrm{CDCl}_{3}+\mathrm{DMSO}-\mathrm{d} 6\right)$ : 7.1-8.5 (m, 9H, Ph, H-5 to H-8), $1.2\left(\mathrm{~s}, 2 \mathrm{H}, \mathrm{CH}_{2}\right) 2.3$ (s, 3H, Me), 5.5 (s, 1H, CH), 8.4 (br, 1H, NH) Pyrazolone. MS: m/z [360] $]^{+}$ Anal. calcd. for $\mathrm{C}_{20} \mathrm{H}_{16} \mathrm{~N}_{4} \mathrm{O}_{3}$ : C, 66.75; $\mathrm{H}, 4.48 ; \mathrm{N}, 15.55$; Found: $\mathrm{C}, 66.78 ; \mathrm{H}, 4.50 ; \mathrm{N}, 15.53$.

6-Bromo-2-methyl-3-\{3-[(3-methyl-5-oxo-4,5-dihydro-1H-pyrazol-1-yl)carbonyl]phenyl\} quinazolin-4(3H)-one (3i). For A Yield: 72\%; For B Yield: 85\% mp 126 ${ }^{\circ} \mathrm{C}$. IR (KBr): 1695 $(\mathrm{C}=\mathrm{O}), 1590(\mathrm{C}=\mathrm{N})$ Quinazoline; 1686, 1699, $(\mathrm{C}=\mathrm{O}), 1315\left(\mathrm{C}-\mathrm{CH}_{3}\right)$ Pyrazolone, 3060 (aromatic $\mathrm{C}-\mathrm{H}), 1540$ (C---C of aromatic ring) 1317 (N-C-N), 547 (C-Br). ${ }^{1} \mathrm{H}-\mathrm{NMR}$ (DMSO-d6): 1.3 (s, 3H, Me), 7.1-7.9 (m, 7H, Ph \& H-5, H-7, H-8), 2.3 (s, 3H, Me), 5.5 (s, 1H, CH), 8.4 (br, 1H, $\mathrm{NH})$ Pyrazolone. MS: m/z [439] $]^{+}$Anal. calcd. for $\mathrm{C}_{20} \mathrm{H}_{15} \mathrm{BrN}_{4} \mathrm{O}_{3}: \mathrm{C}, 60.36 ; \mathrm{H}, 4.09 ; \mathrm{N}, 10.38$; Found: C, 60.38; H, 4.50; N, 15.53 .

2-Methyl-3-\{3-[(3-methyl-5-oxo-4,5-dihydro-1H-pyrazol-1-yl)carbonyl]phenyl\}quinazolin4(3H)-one (3j). For A Yield: 70\%; For B Yield: 89\% mp 195 ${ }^{\circ} \mathrm{C}$. IR (KBr): $1705(\mathrm{C}=\mathrm{O}), 1605$ $(\mathrm{C}=\mathrm{N})$ Quinazoline; 1682, 1685, $(\mathrm{C}=\mathrm{O}), 1307\left(\mathrm{C}-\mathrm{CH}_{3}\right)$ Pyrazolone, 3048 (aromatic $\left.\mathrm{C}-\mathrm{H}\right), 1527$ (C---C of aromatic ring) $1314(\mathrm{~N}-\mathrm{C}-\mathrm{N}) .{ }^{1} \mathrm{H}-\mathrm{NMR}$ (DMSO-d6):1.5 (s, 3H, Me), 7.3-7.7 (m, 8H, $\mathrm{Ph} \& \mathrm{H}-5$ to H-8), 2.5 (s, 3H, Me), 5.7 (s, 1H, CH), 8.3 (br, 1H, NH) Pyrazolone. MS: m/z $[360]^{+}$. Anal. calcd. for $\mathrm{C}_{20} \mathrm{H}_{16} \mathrm{~N}_{4} \mathrm{O}_{3}$ : C, 71.22; H, 5.06; N, 12.78; Found: C, 71.20; H, 5.10; N, 12.80 .

6-Bromo-3-\{3-[(3-methyl-5-oxo-4,5-dihydro-1H-pyrazol-1-yl)carbonyl]phenyl\}-2-phenyl quinazolin-4(3H)-one (3k). For A Yield: 77\%; For B Yield: 90\% mp 275 ${ }^{\circ} \mathrm{C}$. IR (KBr): 1710 $(\mathrm{C}=\mathrm{O}), 1598(\mathrm{C}=\mathrm{N})$ Quinazoline; 1705, 1695, $(\mathrm{C}=\mathrm{O}), 1308\left(\mathrm{C}-\mathrm{CH}_{3}\right)$ Pyrazolone, 3062 (aromatic $\mathrm{C}-\mathrm{H}), 1522$ (C---C of aromatic ring) $1312(\mathrm{~N}-\mathrm{C}-\mathrm{N}), 545$ (C-Br). ${ }^{1} \mathrm{H}-\mathrm{NMR}\left(\mathrm{CDCl}_{3}\right)$ : 7.3-8.2 (m, 12H, 2 x Ph \& H-5, H-7, H-8), 2.3 (s, 3H, Me), 5.9 (s, 1H, CH), 8.2 (br, 1H, NH) Pyrazolone. MS: $\mathrm{m} / \mathrm{z}[501]^{+}$. Anal. calcd. for $\mathrm{C}_{25} \mathrm{H}_{17} \mathrm{BrN}_{4} \mathrm{O}_{3}$ : C, 59.89; H, 3.42; N, 11.18; Found: C, 59.92; $\mathrm{H}, 3.45 ; \mathrm{N}, 11.21$. 
3-\{3-[(3-Methyl-5-oxo-4,5-dihydro-1H-pyrazol-1-yl)carbonyl]phenyl\}-2-phenylquinazolin4(3H)-one (3l). For A Yield: 75\%; For B Yield: 92\% mp 248 ${ }^{\circ}$. IR (KBr): $1695(\mathrm{C}=\mathrm{O}), 1590$ $(\mathrm{C}=\mathrm{N})$ Quinazoline; 1686, 1699, $(\mathrm{C}=\mathrm{O}), 1315\left(\mathrm{C}-\mathrm{CH}_{3}\right)$ Pyrazolone, 3075 (aromatic $\left.\mathrm{C}-\mathrm{H}\right), 1530$ (C---C of aromatic ring) $1318(\mathrm{~N}-\mathrm{C}-\mathrm{N}) .{ }^{1} \mathrm{H}-\mathrm{NMR}\left(\mathrm{CDCl}_{3}\right)$ : 7.2-7.7 (m, 13H, $2 \mathrm{x} \mathrm{Ph} \& \mathrm{H}-5$ to H8), 2.2 (s, 3H, Me), 5.4 (s, 1H, CH), 8.1 (br, 1H, NH) Pyrazolone. MS: m/z [422] ${ }^{+}$. Anal. calcd. for $\mathrm{C}_{20} \mathrm{H}_{18} \mathrm{~N}_{4} \mathrm{O}_{3}$ : C, 71.08; H, 4.29; N, 13.26; Found: C, 71.10 H, 4.27; N, 13.30.

\section{Conclusions}

All compounds were screened for their antifungal against C.albicans ATC and candida krusei and antibacterial against B.subtilis, S.aureus as gram positive and E.coli, P.aeruginosa as gram negative bacteria. Zone of inhibition were measured in millimeters. The antifungal activities of the tested compounds were compared to the standard drug flucanozole $(25-28 \mathrm{~mm})$. DMF was used as solvent. All compounds have shown good activity against candida albicans Table 2. Ampicillin (18-20mm), Amoxicillin (19-21 mm) and penicillin (17-19mm) were used as standard drugs for antibacterial activity. The compounds $\mathbf{3 a}, \mathbf{3 b}, \mathbf{3 e}, \mathbf{3 g}$ and $\mathbf{3 h}$ showed significant activity (11-18 mm) against E.Coli and P. aeruginosa (gram-ve) at $100 \mu \mathrm{g} / \mathrm{mL}$ concentration. The compounds 3a, 3c, 3e, 3i and $\mathbf{3 l}$ showed good activity $(12-19 \mathrm{~mm})$ against S.aureus and B.subtilis (gram $+\mathrm{ve}$ ) at $100 \mu \mathrm{g} / \mathrm{mL}$ concentration. The 3b, 3c, 3e, 3f and 3i showed good to significant activity against C. albicans ATCC 10231 and C. Krusei G03 at $100 \mu \mathrm{g} / \mathrm{mL}$ concentration.

\section{Acknowledgements}

The authors are thankful to Head, Department of Chemistry, Veer Narmad South Gujarat University for providing the research facilities in the organic research laboratory and IR Spectral analysis. The authors are also thankful to CDRI, Lucknow for providing the ${ }^{1} \mathrm{H}$ NMR, mass spectra and elemental analysis. The authors are thankful Dr. Kirity Roy, Quest Institute of Life Sciences, C/O Nicolas Piramal Bombay for providing the antimicrobial testing facility for newly synthesized drugs.

\section{References}

1. Xie, W.; Jin, Y.; Wang, P. G. Chem. Tech. 1999, 29, 23.

2. Verma, R. S. Green Chem. 1999, 1, 43.

3. Satsangi, R. K. Indian Drugs 1979, 17, 79. 
4. Joshi, V.; Chaudhari, R. P. Indian J. Chem. 1987, 26B, 602.

5. Srivastava, V. K.; Gulati, S. S.; Shanker, K. Indian J. Chem. 1987, $26 B, 652$.

6. Gupta, D. P.; Ahmad, S.; Kumar, A.; Shanker, K. Indian J. Chem. 1988, 27B, 1060.

7. Sakai, K.; Nahata. H. Jpn. Kokai Tokyo Koho JP 6351, 329; Chem. Abstr. 1988, 109, 86338.

8. Niementowski, V. J. Prakt. Chem. 1895, 51, 564; Beilstein 24, 143.

9. Gan, Y.; Lu, D.; Liu, J.; Tian, M. Zhongguo yaouri Zazhi 2001, 11(2), 85; Chem. Abstr. 2002, 136, 216696.

10. Turan-Zitouni, G.; Sivaci, M.; Kilic, F. S.; Erol, K. Eur. J. Med. Chem. 2001, 36, 685.

11. Elguero, J. Comprehensive Heterocyclic Chemistry. The structure, reaction, synthesis and uses of heterocyclic compounds, K. T. Potts, Ed.; Pergamon Press: 1984; Vol. 5, p 167.

12. (a) Sharma, R. S.; Bahel, S. C. J. Ind. Chem. Soc. 1982, 59, 877. (b) Shiba, S. A.; ElKhamry, A, A.; Shaban, M. E.; Atia, K. S. Indian J. Chem. 1997, 36B, 361

13. (a) Jung, J. C.; Watkins, E. B.; Avery, M. A. Synth. Commun. 2002, 32, 3767. (b) Scherowsky, G.; Kunda, B. Tetrahedron Lett. 1972, 3169.

14. (a) Kidwai, M.; Saxena, S.; Mohan, R.; Venkataramanan, R. J. Chem. Soc., Perkin Trans. 1 2002, 16, 1845. (b) Kidwai, M.; Rastogi, S.; Venkataramanan, R. Bull. Chem. Soc. Jpn. 2003, 76, 203.

15. Barry, A. L. The Antimicrobial Susceptibility Test, Principle and Practices 1993, 180.

16. Kidwai, M.; Venkataramanan, R. Green Chem. 2001, 3, 278.

17. Kidwai, M.; Saxena, S.; Mohan, R.; Venkataramanan, R. J. Chem. Soc., Perkin Trans. 1 2002, 1, 1845.

18. Leiby, R. W. J. Org. Chem. 1985, 50, 2926.

19. Kidwai, M.; Mohan, R. Org, Proc. Prep. Int. 2003, 35, 426.

20. Kidwai, M.; Rastogi, S.; Mohan, R.; Croatica Chemica Acta 2003, 76, 365.

21. Kidwai, M.; Mohan, R. J. Korean. Chem. Soc. 2004, 48, 177. 